\title{
Image of the month: Rolling stones
}

\author{
Authors: Philipp Pecnik, ${ }^{A}$ Verena Ranft| ${ }^{B}$ and Pius Steiner ${ }^{C}$
}
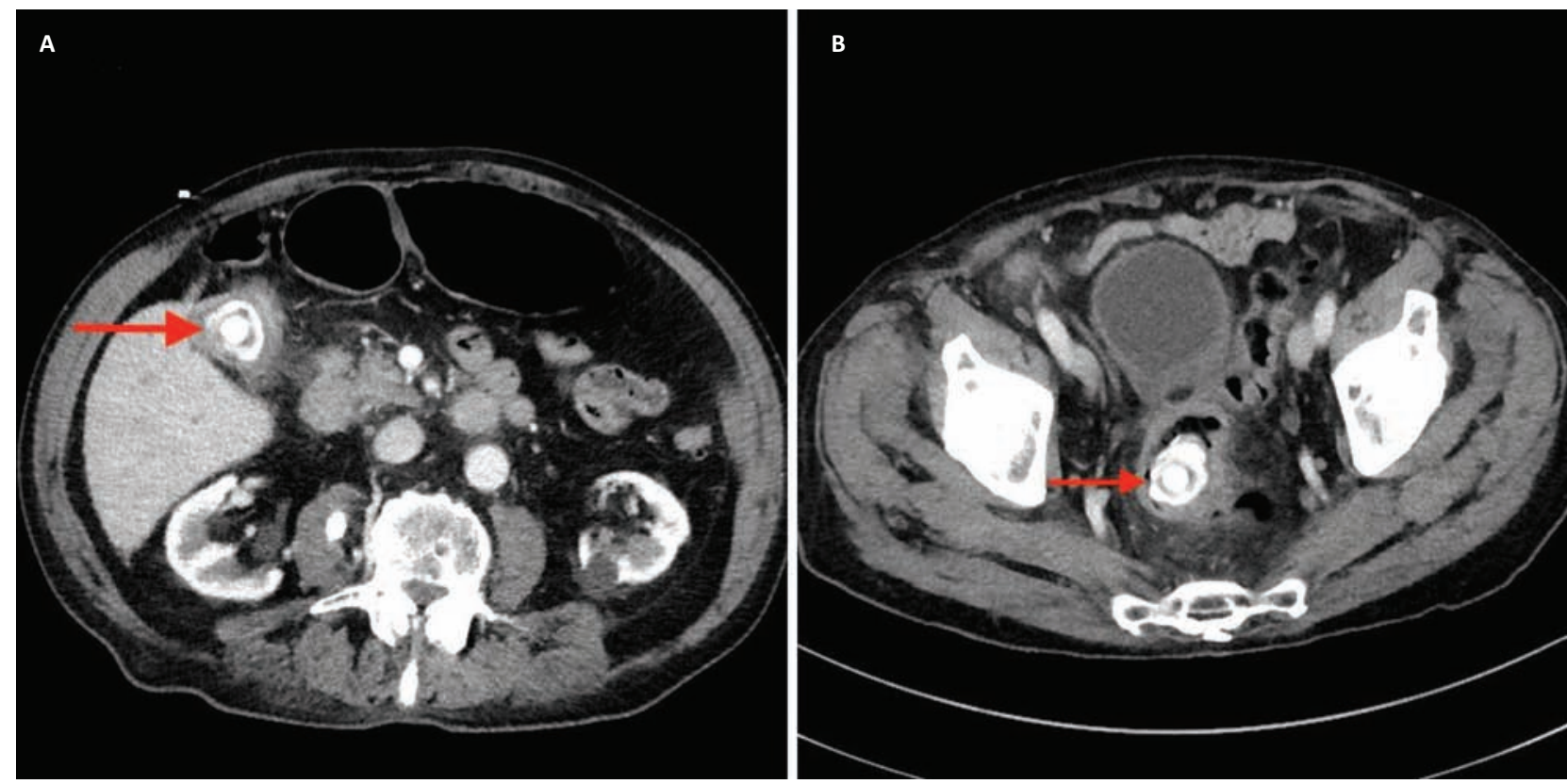

Fig 1. Comparison of a patient's computerised tomography (CT) scans, which were taken 1 month apart. The first CT scan (A) shows chronic gall bladder wall thickening with cholecystolithiasis (red arrow), while the CT scan taken 1 month later (B) reveals relocation of the same gall bladder stone in the sigmoid colon (red arrow).

An 88-year old man with a history of Crohn's disease presented to the emergency department with an acute onset of diffuse abdominal pain since the early morning, accompanied by vomiting and diarrhoea. Physical examination of the abdomen revealed right-sided flank pain, a slightly distended abdomen with tenderness to the right upper quadrant and reduced bowel sounds with absence of rebound. The patient was free of fever or chills.

Initial workup showed elevated inflammatory markers (C-reactive protein $88 \mathrm{mg} / \mathrm{dL}$ (normal range $<5 \mathrm{mg} / \mathrm{dL}$ ), increased pancreatic enzymes (lipase $174 \mathrm{U} / \mathrm{L}$ (normal range $<60 \mathrm{U} / \mathrm{L}$ ); amylase $134 \mathrm{U} / \mathrm{L}$ (normal range $<53 \mathrm{U} / \mathrm{L}$ )) and

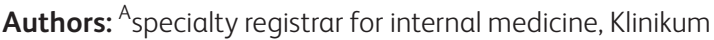
Wels-Grieskirchen, Wels, Austria; ${ }^{{ }}$specialty registrar for general medicine, Klinikum Wels-Grieskirchen, Wels, Austria; ' ${ }^{C}$ consultant in gastroenterology, Klinikum Wels-Grieskirchen, Wels, Austria slightly elevated bilirubin of $1.5 \mathrm{mg} / \mathrm{dL}$ (normal range $0.2-1.0 \mathrm{mg} / \mathrm{dL}$ ). Hepatic enzymes were within normal range. An ultrasound of the abdomen indicated chronic gall bladder wall thickening with cholecystolithiasis and no clear evidence of an extended biliary tree; renal ultrasound showed bilateral narrowing of the parenchyma, but was otherwise unremarkable.

Administration of intravenous analgetics led to symptom relief.

The following night the patient experienced massive abdominal pain but failed to report this incidence to the medical staff. During the medical round the next day the patient mentioned the nightly discomfort. Given the patient's history, symptoms and the present laboratory results, acute computerised tomography (CT) scan was initiated and compared with a CT scan that was taken 1 month prior because of similar symptoms (Fig 1). The CT scan revealed an acute perforation of the gallbladder into the transverse colon indicated by the passing of a large gallstone, 
which was initially located in the fundus of the gall bladder (Fig 1A, red arrow) and now situated in the sigmoid colon (Fig 1B, red arrow). Also, aerobilia was described, located in the ductal tree. Conceivably, the acute abdominal pain was associated with inflammation and perforation of the gallbladder.

Soon after he underwent surgery and laparotomy with cholecystectomy and colon segment resection was performed. The postoperative clinical course was prolonged because of insufficient wound healing. The patient was discharged from our surgical ward on the 21st postoperative day in a stable status.

\section{Conflicts of interest}

The authors have no conflicts of interest to declare.

\section{Acknowledgement}

The patients' consent was obtained for publication of the clinical details and images in this article.

Address for correspondence: Dr Philipp Pecnik, Department of Internal Medicine, Klinikum Wels-Grieskirchen, Grieskirchnerstrasse 42, 4600 Wels, Austria.

Email: pecnikphilipp@gmail.com 